\title{
SPITZER CHARACTERIZATION OF DUST IN THE IONIZED MEDIUM OF THE LARGE MAGELLANIC CLOUD
}

\author{
Déborah Paradis ${ }^{1}$, Roberta Paladini $^{1}$, Alberto Noriega-Crespo $^{1}$, Guilaine Lagache $^{2}$, Akiko Kawamura $^{3}$, \\ TOSHIKAZU ONISHI ${ }^{4}$, AND Yasuo FuKuI ${ }^{3}$ \\ ${ }^{1}$ Spitzer Science Center, California Institute of Technology, Pasadena, CA 91125, USA \\ 2 Institut d'Astrophysique Spatiale, 91405 Orsay, France \\ ${ }^{3}$ Department of Astrophysics, Nagoya University, Chikusa-ku, Nagoya 464-8602, Japan \\ ${ }^{4}$ Department of Physical Science, Osaka Prefecture University, Gakuen 1-1, Sakai, Osaka 599-8531, Japan \\ Received 2010 December 17; accepted 2011 April 4; published 2011 June 8
}

\begin{abstract}
A systematic investigation of dust emission associated with ionized gas has so far been performed only in our Galaxy and for wavelengths longer than $60 \mu \mathrm{m}$. Newly available Spitzer data now offer the opportunity to carry out a similar analysis in the Large Magellanic Cloud (LMC). By cross-correlating Spitzer Surveying the Agents of a Galaxy's Evolution (SAGE) data with the Australia Telescope Compact Array/Parkes H I $21 \mathrm{~cm}$ data, the NANTEN ${ }^{12} \mathrm{CO}(J=1-0)$ data, and both the Southern H-Alpha Sky Survey Atlas $\mathrm{H} \alpha$ and the Parkes $6 \mathrm{~cm}$ data, we investigate the physical properties of dust associated with the different phases of the gas (atomic, molecular, and ionized). In particular, we study the presence and nature of dust from 3.6 to $160 \mu \mathrm{m}$ and for various regimes of ionized gas, spanning emission measures from $\sim 1 \mathrm{pc} \mathrm{cm}^{-6}$ (diffuse component) to $\sim 10^{3} \mathrm{pc} \mathrm{cm}^{-6}$ (H II regions). Using a dust emission model and testing our results with several radiation field spectra, we show that dust in ionized gas is warmer than dust associated with other phases (atomic and molecular). We also find a decrease of the polycyclic aromatic hydrocarbon (PAH) relative abundance with respect to big grains, as well as an increase of the near-infrared (NIR) continuum. These three results (i.e., warmer temperature, decrease of PAH abundance, and increase of the NIR continuum) are found consistently for all regimes of ionized gas. On the contrary, the molecular phase appears to provide favorable conditions for the survival of PAHs. Furthermore, the very small grain relative abundance tends to increase in the ionized phase, especially in bright $\mathrm{H}$ II regions. Last but not least, our analysis shows that the emissivity of dust associated with ionized gas is lower in the LMC than in our Galaxy and that this difference is not accounted for by the lower metallicity of the LMC.
\end{abstract}

Key words: dust, extinction - H II regions - Magellanic Clouds

Online-only material: color figure

\section{INTRODUCTION}

Detecting dust emission associated with the diffuse ionized phase is a complex task due to two main competing factors. First, large grains are expected to be selectively destroyed in the warm ionized medium (WIM) due to the passage of multiple shock fronts which would reduce their emission. In addition, along most lines of sight (LOSs), the diffuse ionized gas is likely mixed with the other phases of the gas (atomic and molecular), and its contribution is not expected to be dominant at any latitude.

Because of these difficulties, contradictory results concerning the presence of dust in the WIM have been reported in the literature. Lagache et al. (1999), looking at the high Galactic latitude residuals of the IR/H I correlation between 100 and $1000 \mu \mathrm{m}$, claim a statistical detection. In particular, by adopting an emissivity spectral index of 2 , they find an emissivity law in the atomic and ionized gas phases of, respectively, $\tau / N_{\mathrm{H}}=8.7 \pm 0.910^{-26}(\lambda / 250 \mu \mathrm{m})^{-2} \mathrm{~cm}^{-2}$ and $\tau / N_{\mathrm{H}}=$ $1.0 \pm 0.210^{-25}(\lambda / 250 \mu \mathrm{m})^{-2} \mathrm{~cm}^{-2}$, with $T_{\mathrm{HI}}=17.5 \mathrm{~K}$ and $T_{\mathrm{H}+}=20 \mathrm{~K}$. Following this preliminary investigation, Lagache et al. (2000) extend their analysis and carry out a decomposition of the far infrared (FIR) emission using WHAM (Reynolds et al. 1998) $\mathrm{H} \alpha$ data as a tracer of the ionized medium. The result of this study confirms the early detection, with an emissivity law for the ionized phase of $\tau / N_{\mathrm{H}}=1.110^{-25}(\lambda / 250 \mu \mathrm{m})^{-2} \mathrm{~cm}^{-2}$, for a fixed temperature of $17.2 \mathrm{~K}$ for both the atomic and ionized phases. At low Galactic latitudes, Paladini et al. (2007) derive dust emissivities associated with the atomic, molecular, and ionized gas phases, using an inversion method applied to IRAS (Neugebauer et al. 1984), DIRBE (Hauser 1993), Archeops (Benoît et al. 2002), and WMAP (Bennett et al. 2003) data. According to their analysis, the average dust temperatures in the atomic and molecular phases are comparable, and of order of 19.8 and $19.2 \mathrm{~K}$, respectively, while dust in the ionized phase is significantly warmer, $T_{\mathrm{H}+} \simeq 26.7 \mathrm{~K}$. At the same time, Odegard et al. (2007) looking at the DIRBE/WHAM correlation in five selected high-latitude Galactic regions find, contrary to Lagache et al. (2000), that the $\mathrm{H} \alpha$-correlated dust component is negligible in all cases.

Despite the observational caveats, assessing the presence of dust in the ionized gas phase is very important, given that the optical properties of dust grains can vary with the properties of the local environment. To date, no clear-cut indication of such a behavior has been found though. Paradis et al. (2011) perform a statistical study of all the molecular clouds in the Large Magellanic Cloud (LMC) and do not find any systematic change in dust abundances nor in dust temperature between the molecular and the atomic phases. On the contrary, Stepnik et al. (2003) and Paradis et al. (2009a), by looking at nearby Galactic clouds, do report evidence of this kind of variations. In the ionized medium, due to the proximity of the ionized gas to UV sources such as O and B stars and the cumulative effect of shock fronts, changes in the optical dust properties are indeed expected. For instance, depletion of polycyclic aromatic 
hydrocarbons (PAHs) has been observed toward individual H II regions (Everett \& Churchwell 2010; Watson et al. 2009; Contursi et al. 2007; Peeters et al. 2005; Povich et al. 2007). However, this phenomenon does not appear to be systematic, and its origin is still poorly understood.

In this paper, we present a systematic investigation of the properties of dust associated with the ionized gas in the LMC. The LMC, located at a distance of $\sim 50 \mathrm{kpc}$ (Feast 1999; van Leeuwen et al. 2007), has an advantageous almost face-on viewing angle. At $-32^{\circ}$ latitude, the contamination along the LOS is expected to be small compared to the plane of the Galaxy. For this galaxy, Bernard et al. (2008) carried out a cross-correlation study of the combined Spitzer SAGE (Meixner et al. 2006) and IRAS data, with H I and CO data. That work allowed us to characterize (in terms of emissivities, abundances, etc.) the properties of dust mixed with the atomic and molecular media, as well as to point out the existence of an excess of emission at $70 \mu \mathrm{m}$. Interestingly, Bernard et al. (2008) showed that this excess appears to be spatially correlated with both the atomic and the diffuse $\left(I_{\mathrm{H}_{\alpha}}<200 R\right)$ ionized gas.

The present study builds on and expands the analysis described in Bernard et al. (2008), by including the ionized medium in the cross-correlation and by encompassing different physical regimes of this phase of the gas, from the most tenuous environments (diffuse medium) to the densest ones (H II regions).

The paper is organized as follows. Section 2 summarizes the data we used. In Section 3, we describe the decomposition method as well as the dust emission modeling. We present our main results in Section 4. Concluding remarks are provided in Section 5 .

\section{OBSERVATIONS}

\subsection{IR Data}

Along the lines of Bernard et al. (2008), we use, as a tracer of dust emission from the NIR to the FIR, the Spitzer SAGE data. The data span the wavelength range $3.6 \mu \mathrm{m}$ to $160 \mu \mathrm{m}$, with an angular resolution from $\simeq 2^{\prime \prime}$ to $40^{\prime \prime}$, respectively.

These Spitzer data are combined with the 12 and $100 \mu \mathrm{m}$ infrared interferometer spectrometer (IRIS) data (Improved Reprocessing of the IRAS Survey; Miville-Deschênes \& Lagache 2005) characterized by an angular resolution close to $4^{\prime}$. The inclusion of the IRAC $8 \mu \mathrm{m}$ and IRIS $12 \mu \mathrm{m}$ data allows us to take into account the contribution from the most prominent PAH features, i.e., at 7.7 and $11.3 \mu \mathrm{m}$, while with MIPS 24 and $70 \mu \mathrm{m}$ the bulk of the emission from very small grains (VSGs) is incorporated. In addition, the $100 \mu \mathrm{m}$ IRIS data point, combined with the MIPS $160 \mu \mathrm{m}$ measurement, provides the necessary leverage for the derivation of the equilibrium temperature of the big grains (BGs).

\subsection{Gas Tracers}

\subsubsection{H I Data}

As a tracer of atomic gas, we use the Kim et al. (2003) $21 \mathrm{~cm}$ map, which is a combination of interferometric data obtained with the Australia Telescope Compact Array (ATCA), at $1^{\prime}$ angular resolution, and the Parkes antenna, at 15'3 angular resolution (Staveley-Smith et al. 2003). Assuming the gas is optically thin, the column density can be derived using the relation

$$
N_{\mathrm{HI}}=X_{\mathrm{HI}} W_{\mathrm{HI}},
$$

where $W_{\mathrm{H}}$ is the $\mathrm{H}$ integrated intensity map and the conversion factor $X_{\mathrm{H}_{\mathrm{I}}}$ is taken equal to $1.82 \times 10^{18} \mathrm{H} / \mathrm{cm}^{2} /(\mathrm{K} \mathrm{km} / \mathrm{s})$ (Spitzer 1978). The integrated map was built by summing up all the velocity channels from 190 to $386 \mathrm{~km} \mathrm{~s}^{-1}$. For the purpose of estimating and subtracting the Galactic foreground contribution to each IR wavelength, we use a Galactic H i column density map constructed by Staveley-Smith et al. (2003). More details on this procedure can be found in Bernard et al. (2008).

\subsubsection{CO Data}

The ${ }^{12} \mathrm{CO}(J=1-0)$ survey obtained with the $4 \mathrm{~m}$ radio NANTEN telescope of Nagoya University at Las Campanas Observatory, Chile (see Fukui et al. 2008) is adopted as a tracer of the molecular gas. In particular, we make use of the second NANTEN survey which, characterized by an angular resolution of $2: 6,{ }^{5}$ gave evidence of the existence of 272 individual molecular clouds in that galaxy (Fukui et al. 2008).

We compute the molecular column densities using the relation

$$
N_{\mathrm{H}_{2}}=X_{\mathrm{CO}} W_{\mathrm{CO}},
$$

where $W_{\mathrm{CO}}$ is the $\mathrm{CO}$ integrated intensity map, generated by integrating the original $\mathrm{CO}$ cube over the $200 \mathrm{~km} \mathrm{~s}^{-1}<v_{\mathrm{lsr}}<$ $320 \mathrm{~km} \mathrm{~s}^{-1}$ velocity range. Fukui et al. (2008) derived the $X_{\mathrm{CO}}$ conversion factor assuming that each cloud is at virial equilibrium. They found an average value for all the clouds of $X_{\mathrm{CO}}=(7 \pm 2) \times 10^{20} \mathrm{H}_{2} / \mathrm{cm}^{2} /\left(\mathrm{K} \mathrm{km} \mathrm{s}^{-1}\right)$. From cloud to cloud, values vary from $1.6 \times 10^{20}$ to $4.2 \times 10^{21} \mathrm{H}_{2} / \mathrm{cm}^{2} /(\mathrm{K} \mathrm{km} / \mathrm{s})$. Their average value is larger than that estimated by Hughes et al. (2010) of $4.7 \times 10^{20} \mathrm{H}_{2} / \mathrm{cm}^{2} /(\mathrm{K} \mathrm{km} / \mathrm{s})$, using the Mopra telescope data, at a resolution of $33^{\prime \prime}$. This difference is due to the fact that low $\mathrm{CO}$ brightness clouds have been excluded from the MOPRA survey, and the $X_{\mathrm{CO}}$ value has been deduced from a smaller number of clouds compared to NANTEN observations. In this work, in order to be consistent with our previous studies (e.g., Paradis et al. 2011), we use the $X_{\mathrm{CO}}$ values determined for each individual cloud by Fukui et al. (2008). For clouds for which the virial mass could not be derived, we adopt the LMC average value of $X_{\mathrm{CO}}=(7 \pm 2) \times 10^{20} \mathrm{H}_{2} / \mathrm{cm}^{2} /\left(\mathrm{K} \mathrm{km} \mathrm{s}^{-1}\right)$. For a comprehensive tabulation of $X_{\mathrm{CO}}$ values, we refer the reader to Paradis et al. (2011, see their Table 5).

\subsubsection{Ho and Radio Data}

The warm ionized gas, characterized by an electron temperature of the order of $T_{\mathrm{e}} \simeq 10^{4} \mathrm{~K}$, emits both recombination lines and radio free-free continuum. Among the observed recombination lines, the $\mathrm{H} \alpha$ line is the brightest and is due to a transition of the Balmer line series at $6562.81 \AA$. As for free-free emission, this consists of free electrons being scattered and decelerated by their encounters with ions. $\mathrm{H} \alpha$ and radio templates are available for the LMC and both tracers are used in our analysis to assure an independent cross-check of the results. For the $\mathrm{H} \alpha$, we use the Southern H-Alpha Sky Survey Atlas (SHASSA; Gaustad et al. 2001) data set which covers the southern hemisphere $\left(\delta<15^{\circ}\right)$, at an angular resolution of 0.8 , and with a sensitivity of 2 Rayleigh pixel ${ }^{-1}\left(1 R=10^{6}\right.$ photons $\left.\mathrm{cm}^{-2} \mathrm{~s}^{-1}\right)$. Following Lagache et al. (1999), if we assume that the electron density $n_{\mathrm{e}}$ is constant along each LOS, the $\mathrm{H}^{+}$column density can be derived using the relation

$$
\frac{N_{\mathrm{H}}^{\mathrm{H}^{+}}}{\mathrm{Hcm}^{-2}}=1.37 \times 10^{18} \frac{I_{\mathrm{H} \alpha}}{R} \frac{n_{\mathrm{e}}}{\mathrm{cm}^{-3}},
$$

\footnotetext{
5 About $40 \mathrm{pc}$ at the distance of the LMC.
} 
where $1 R=2.25 \mathrm{pc} \mathrm{cm}^{-6}$ for $T_{\mathrm{e}}=8000 \mathrm{~K}$ (see, for instance, Dickinson et al. 2003). For $n_{\mathrm{e}}$, we adopt different values for different regimes of the ionized gas, as described in Section 3.1.

To trace the radio free-free emission, we use observations obtained with the Parkes $64 \mathrm{~m}$ telescope at $4.75 \mathrm{GHz}$ (see Filipovic et al. 1995 for a complete description of the Parkes survey). At that frequency the beam size is 4.9 , but after correcting for scanning effects, the effective beam size results to 5'6.

In order to compare the radio and $\mathrm{H} \alpha$ maps and compute gas column densities for the radio map, we transform the radio emission to $\mathrm{H} \alpha$ units, using Equation (11) in Dickinson et al. (2003):

$$
\frac{T_{\mathrm{b}}}{I_{\mathrm{H} \alpha}}=8.396 \times 10^{3} a \times v_{\mathrm{GHz}}^{-2.1} T_{4}^{0.667} 10^{0.029 / T_{4}}(1+0.08),
$$

where $T_{\mathrm{b}}$ (brightness temperature) is in $\mu \mathrm{K}$ and $I_{\mathrm{H} \alpha}$ in Rayleigh. $T_{4}$ is $T_{\mathrm{e}}$ in units of $10^{4} \mathrm{~K}$, while the factor $a$ depends on the frequency and the electron temperature (see Table 3 in Dickinson et al. 2003) and $v_{\mathrm{GHz}}$ is the frequency in GHz. For $T_{\mathrm{e}}=8000 \mathrm{~K}$ $\left(T_{4}=0.8\right), a$ is close to unity. To apply Equation (4), we first convert the original units of the Parkes map $\left(\mathrm{mJy} \mathrm{beam}^{-1}\right)$ into Jy, and these into $T_{\mathrm{b}}$ using the expression

$$
T_{\mathrm{b}}=\frac{S_{4.75 \mathrm{GHz}} c^{2}}{2 k v^{2}}
$$

with $S_{4.75 \mathrm{GHz}}$ the total flux density at $4.75 \mathrm{GHz}, c$ the speed of light, $v$ the frequency, and $k$ the Boltzmann constant. Once the Parkes map has been converted into $I_{\mathrm{H} \alpha}$ units, the $\mathrm{H}^{+}$ column density can be computed by applying Equation (3). The sensitivity of the Parkes telescope is $8 \mathrm{mJy} \mathrm{beam}^{-1}$, also equivalent to $28 R$, i.e., significantly lower than the sensitivity threshold of the SHASSA map. This implies, as we will also see in Section 3.2, that while the SHASSA map will be used as a tracer of the ionized gas for all types of environmental conditions (i.e., from the diffuse to the dense ionized medium), the Parkes map will only be used to probe the densest/brightest regions.

For the pixels corresponding to bright $\mathrm{H}$ II regions, we have performed a comparison of the radio Parkes map, converted into $\mathrm{H} \alpha$ emission $\left(I_{\mathrm{H} \alpha}\right)$, with the SHASSA map. The comparison highlights that, for $88 \%$ of the pixels, the $\mathrm{H} \alpha$-Parkes data are higher than the SHASSA one. The median value of the ratio between the two data sets is 1.6. To explain such a discrepancy, we invoke extinction as a source of attenuation in the SHASSA map. In this respect, we remind the reader that $\mathrm{H} \alpha$ measurements are affected by dust absorption, while radio data provide an uncontaminated observational window into the ionized gas. In the LMC, extinction is usually considered rather low. For instance, Imara \& Blitz (2007) and Dobashi et al. (2008) quote an average $A(V)$ of $0.3 \mathrm{mag}$. In our analysis, the discrepancy factor of 1.6 would induce an extinction value in the SHASSA map of $0.51 \mathrm{mag}$ in bright $\mathrm{H}$ II regions. Following $\mathrm{O}^{\prime}$ Donnell (1994), Dickinson et al. (2003) estimate the absorption in $\mathrm{H} \alpha$ as $A(\mathrm{H} \alpha)=0.81 A(V)$. This result is obtained using an extinction curve for the Milky Way. However, as shown by Gordon et al. (2003), the extinction curve in the LMC is very similar to the Galactic one. Therefore, the estimate above also applies here. In that case, the corresponding extinction of LMC bright $\mathrm{H}_{\text {II }}$ regions in the visible is $A(V)=0.63 \mathrm{mag}$. This value is slightly higher than the extinction estimated using the LMC $A(V)$ map constructed by Dobashi et al. (2008), and weighted by the $\mathrm{H} \alpha$

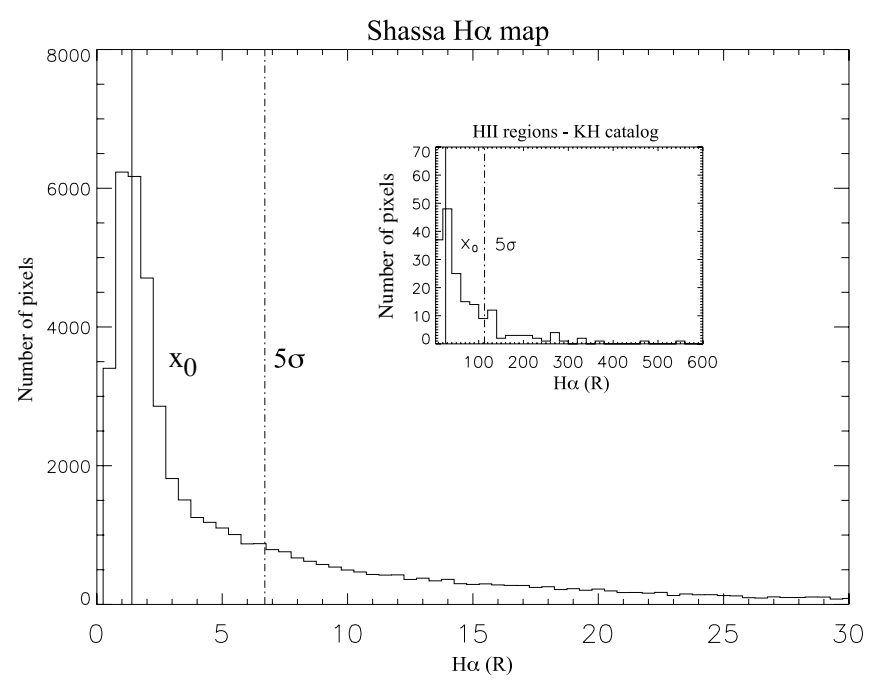

Figure 1. Pixel intensity distribution of the SHASSA H $\alpha$ map. Values on the $x$-axis have been truncated to $30 R$ to show the bulk of the distribution. Overlaid are (solid line) the mean $\left(x_{0}\right)$ and (dashed line) $5 \sigma$ values. Inset panel: pixel intensity distribution for the KH86 H II regions in the SHASSA map. The mean value and $5 \sigma$ limits are also shown.

emission $\left(\sum A(V) I_{\mathrm{H} \alpha} / \sum I_{\mathrm{H} \alpha} \simeq 0.57 \mathrm{mag}\right.$ ) over the same $\mathrm{H}_{\mathrm{II}}$ regions. It is unlikely that bright $\mathrm{H}$ II regions are all confined behind dust clouds, which may indicate that the extinction could have been underestimated when constructing the LMC $A(V)$ map from star counts, as already suggested in Paradis et al. (2011). In the following, we therefore expect differences of about a factor of 1.6 when comparing results using SHASSA and Parkes data (see Section 4.1.3).

All the data used for the analysis (IR, H I, $\mathrm{CO}, \mathrm{H} \alpha$ ) have been degraded to the lowest angular resolution of the Parkes 4.75 GHz map, using a Gaussian kernel with an FWHM of 5'.6. After being convolved, the maps have been reprojected onto the same grid, with a $2^{\prime}$ pixel size. Finally, point-source subtraction has been applied to all the IR maps (see Bernard et al. 2008 for the description of the map processing).

\section{DECOMPOSITION OF THE IR EMISSION}

\subsection{Regimes of the Ionized Gas}

We consider three regimes of the ionized gas, i.e., from diffuse $\mathrm{H}^{+}$to bright $\mathrm{H}$ II regions, and for each regime we investigate the dust properties independently, due to potentially intrinsic variations in dust composition, evolution, and physics of the grains. To define each regime, we set three thresholds in $\mathrm{H} \alpha$ emission intensity using the SHASSA map, convolved to 5'.6 resolution, and the catalog of $\mathrm{H}$ II regions in the LMC compiled by Kennicutt \& Hodge (1986, hereafter KH86).

We start by analyzing the pixel intensity distribution in the SHASSA map (see Figure 1). The mean $\left(\overline{I_{\mathrm{H} \alpha}}\right)$ of the distribution is $1.4 R$, with a dispersion $(\sigma)$ of $1.1 R$ and a minimum of $0.4 R$, which is the sensitivity of the SHASSA data for the 5'.6 angular resolution and the $2^{\prime}$ pixel size of the map. About $99 \%$ of the pixels lies in the range $0.4 R<I_{H \alpha}<6.7 R$, corresponding to $\overline{I_{\mathrm{H} \alpha}} \pm 5 \sigma$. We note that none of the sources in the KH86 catalog are located in regions of the SHASSA map where the $\mathrm{H} \alpha$ intensity is within this range (see Figure 2), and therefore, we adopt it to define the diffuse ionized gas regime.

For $I_{\mathrm{H} \alpha}>6.7 R$, we look, in the SHASSA map, at the intensity distribution of the pixels which correspond to the location of the 

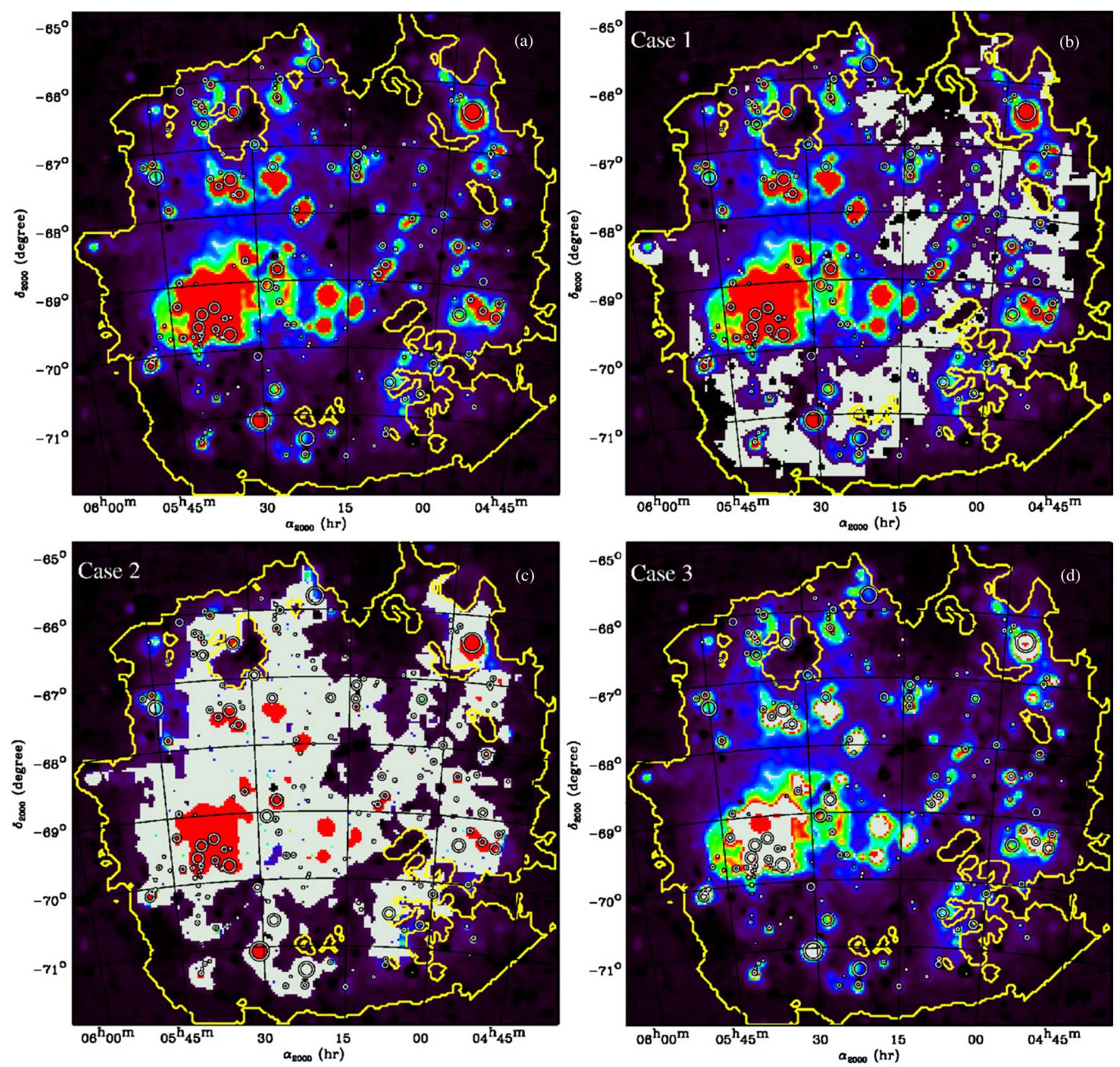

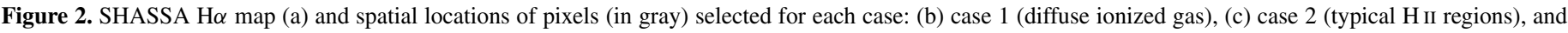
(d) case 3 (bright H II regions). The overlaid symbols show the H II regions (Kennicutt \& Hodge 1986). The range of the maps is [-8; 100] $R$.

(A color version of this figure is available in the online journal.)

H II regions in the KH86 list. To select the pixels, we use, for each cataloged source, its coordinates and measured angular diameter. The $\mathrm{H} \alpha$ intensity distribution obtained by this process is shown in Figure 1 (inset panel). This is characterized by a mean value of $26.3 R$, with a dispersion of $17.4 R$. In this case, $99 \%$ of the pixels lie in the range $6.7 R<I_{\mathrm{H} \alpha}<113.3 R$, and such a range is used to define what, in this context, we denote as typical $\mathrm{H}$ II regions.

For $I_{\mathrm{H} \alpha}>113.3 R$, we have a few very bright H II regions which are listed in the KH86 catalog. We set an upper limit of $1000 R$ to exclude extremely bright sources, such as 30 Doradus, which-due to their complexity-would require a dedicated analysis. The range $113.3 R<I_{\mathrm{H} \alpha}<1000 R$ hence identifies the third regime.
In summary, we have three distinct regimes/cases which we will be tackled in our analysis:

1. case 1: $0.4 R<I_{\mathrm{H} \alpha}<6.7 R$ (diffuse ionized gas);

2. case 2: $6.7 R<I_{\mathrm{H} \alpha}<113.3 R$ (typical H II regions);

3. case 3: $113.3 R<I_{\mathrm{H} \alpha}<1000 R$ (very bright $\mathrm{H}$ II regions).

For each regime, we need to adopt a characteristic $n_{\mathrm{e}}$ in order to compute the $\mathrm{H}^{+}$column density from Equation (3). For the diffuse component, no estimate is available from the literature. We have made an attempt to derive this value directly from the SHASSA map. For this purpose, we first compute, for each pixel in the range $0.4 R<I_{\mathrm{H} \alpha}<6.7 R$, the emission measure (EM), by applying Equation (4) in Dickinson et al. (2003) and assuming $T_{\mathrm{e}}=8000 \mathrm{~K}$. To derive the rms electron density from 
the average $\mathrm{EM}$ so computed $\left(\overline{\mathrm{EM}}=5.3 \pm 0.1 \mathrm{pc} \mathrm{cm}^{-6}\right)$, we need to assume a linear scale of the emitting ionized medium. Our 5'.6 SHASSA map has a pixel size of 2'. At a distance of $50 \mathrm{kpc}$, this corresponds to a linear size of $\sim 30 \mathrm{pc}$. However, such a value is very likely a severe underestimate of the actual vertical extent of the diffuse ionized gas. In fact, KH86 have analyzed the distribution in linear diameters for their cataloged $\mathrm{H}$ II regions, and obtained that the largest sources in the sample have a size of about $400 \mathrm{pc}$. In the hypothesis that the diffuse gas extends far beyond the layer of discrete $\mathrm{H}$ II regions, if we denote with $d_{\mathrm{H}^{+}}$the vertical extent of $\mathrm{H}^{+}$and take $d_{\mathrm{H}^{+}}=500 \mathrm{pc}$, we obtain an average electron density $n_{\mathrm{e}_{1}}$ of $0.10 \pm 0.08 \mathrm{~cm}^{-3}$. This value is close to what has been derived for our Galaxy, i.e., $n_{\mathrm{e}} \sim 0.03-0.08 \mathrm{~cm}^{-3}$, for an estimated scale height ranging between 1000 and 1800 pc (Haffner et al. 2009). If, instead of $500 \mathrm{pc}$, we assume $d_{\mathrm{H}^{+}}=1000 \mathrm{pc}$, we retrieve $n_{\mathrm{e}_{1}}=0.07 \pm$ $0.06 \mathrm{~cm}^{-3}$, matching the Galactic value. Despite such a result, given the uncertainty in $d_{\mathrm{H}^{+}}$for the LMC, we choose to adopt the average value derived from Galactic measurements. Therefore, for case 1 , we take $n_{\mathrm{e}_{1}}=0.055 \mathrm{~cm}^{-3}$.

For cases 2 and 3, we compute EM, for each $\mathrm{H}$ II region in the KH86 catalog by applying Equation (4) in Dickinson et al. (2003). For each source, we use the quoted $\mathrm{H} \alpha$ flux and we set $T_{\mathrm{e}}=8000 \mathrm{~K}$. The measured $\mathrm{H} \alpha$ flux is converted into Rayleighs, and the intensity cuts at $113.3 R$ and $1000 R$ for, respectively, cases 2 and 3 are applied. The averaged EMs for the two cases $\left(\overline{E M_{2}}=183.8 \pm 17.8 \mathrm{pc} \mathrm{cm}^{-6}, \overline{E M_{3}}=1322.4 \pm\right.$ $167.7 \mathrm{pc} \mathrm{cm}^{-6}$ ) are then used to derive an rms electron density. Following this procedure, we obtain $n_{\mathrm{e}_{2}}=1.52 \pm 0.44 \mathrm{~cm}^{-3}$ and $n_{\mathrm{e}_{3}}=3.98 \pm 2.05 \mathrm{~cm}^{-3}$, i.e., 20 and 80 times larger than the diffuse component.

\subsection{Method}

In the LMC, Bernard et al. (2008) showed evidence of the existence of a large FIR excess with respect to the atomic, molecular, and ionized gas phases, which they attributed to either molecular gas with no associated $\mathrm{CO}$ emission or to optically thick H I. They showed that this component is spatially correlated with H I. Paradis et al. (2011) performed a statistical analysis of LMC molecular clouds and showed that if the FIR excess was not taken into account in the IR correlations, the dust abundance in the atomic phase would be systematically overestimated by a factor 1.7 (see their Section 3). Following Paradis et al. (2011), we decompose the IR emission at each wavelength and for each regime, as a function of the atomic, molecular, and ionized gas column densities:

$$
I(\lambda)=a\left(N_{\mathrm{H}}^{\mathrm{H}}+N_{\mathrm{H}}^{\mathrm{X}}\right)+b N_{\mathrm{H}}^{\mathrm{CO}}+c N_{\mathrm{H}}^{\mathrm{H}+}+d
$$

with $N_{\mathrm{H}}^{\mathrm{X}}$ the column density in the FIR excess component. In the following, we refer to the sum of the atomic and FIR excess components as the atomic phase. Despite this working hypothesis, it is important to keep in mind that the FIR excess could be molecular in nature, instead of atomic, and associated with warm $\mathrm{H}_{2}$ gas (Planck Collaboration 2011). The parameters $a, b, c$, denoting the emissivities associated with each phase of the gas at a given wavelength, and the constant $d$ are determined using regression fits. To remove any possible offsets in the data, we subtract a background in all the maps. The background is computed as the median over a common area corresponding to the faintest $10 \%$ of the $\mathrm{H}$ I data. This step also ensures that we have a null IR emission for a null column density. The $\mathrm{H} \alpha$ data are used for all regimes of ionized gas described in the previous section, while, due to the limited sensitivity of the 4.75 GHz map, we can only use the radio data for case 3. As a consequence, a direct cross-check of the results obtained by adopting both tracers will only be possible for this case.

Although case 3 corresponds to bright $\mathrm{H}$ iI regions for which, presumably, most of the radio emission is due to thermal free-free, we might yet have a residual non-thermal contamination associated with synchrotron radiation. To investigate the synchrotron contamination, we complement the $4.75 \mathrm{GHz}$ Parkes data with the $1.4 \mathrm{GHz}$ data, obtained with the same telescope. Both maps are smoothed to the common resolution of 16'.6, characterizing the $1.4 \mathrm{GHz}$ data. Following Niklas et al. (1997), we estimate the fraction of emission contributed by free-free as

$$
\frac{S_{4.75}}{S_{1.4}}=f_{\mathrm{th}}\left(\frac{v_{4.75}}{v_{1.4}}\right)^{-\alpha_{\mathrm{ff}}}+\left(1-f_{\mathrm{th}}\right)\left(\frac{\nu_{4.75}}{v_{1.4}}\right)^{-\alpha_{\mathrm{sync}}},
$$

where $f_{\text {th }}$ is the thermal fraction at $4.75 \mathrm{GHz}, S_{4.75}$ and $S_{1.4}$ are the total flux densities at 4.75 and $1.4 \mathrm{GHz}$, and $\alpha_{\mathrm{ff}}$ and $\alpha_{\text {sync }}$ are the free-free and synchrotron spectral indices. Following Paladini et al. (2005) and Hughes et al. (2006), we take $\alpha_{\mathrm{ff}}=$ 0.1 . The spectral index of synchrotron radiation is known to present large spatial variations. In our Galaxy, it ranges between 0.6 in star-forming complexes to 1.2 in the interarm regions. If, in Equation (7), we adopt a fixed value of $\alpha_{\text {sync }}=0.6$, we derive an average thermal fraction in the LMC of 0.85 . This value is probably an underestimate due to the fact that, while $\alpha_{\mathrm{ff}}=0.1$ is a good approximation for frequencies less than or equal to a few $\mathrm{GHz}$ and $T_{\mathrm{e}}=8000 \mathrm{~K}$, the actual free-free spectral index is a weak function of both frequency and electron temperature (Bennett et al. 1992). Moreover, H II regions span a wide range of $T_{\mathrm{e}}$ values. If we compare our result with similar attempts found in the literature, we see that, for instance, Meinert et al. (1993) tried to separate the thermal and non thermal component in the radio emission of the LMC, using $\mathrm{H} \alpha$ and FIR data, at a resolution of $51^{\prime}$. At $4.75 \mathrm{GHz}$, they obtained a thermal fraction of 0.59 . However, their result applies to both the diffuse medium and discrete $\mathrm{H}$ II regions, while we only consider $\mathrm{H}$ II regions.

In order to take the synchrotron contamination into account, we multiply the $4.75 \mathrm{GHz}$ radio map by the derived thermal fraction equal to 0.85 . Results of the correlations for each phase and regime of the gas are presented in Figure 3 and in Table 1.

\subsection{Modeling}

\subsubsection{Single Radiation Field Along The LOS}

From Equation (6) we obtain the emissivities associated with each phase of the gas for each wavelength $\lambda$ in the 3.6-160 $\mu \mathrm{m}$ range, and with these values we build the corresponding spectral energy distributions (SEDs). The SEDs are modeled with DustEM (Compiègne et al. 2008, 2010). The DustEM code is an updated version of the Désert et al. (1990) model. The code allows us to constrain the mass abundances $\left(Y_{\mathrm{BG}}, Y_{\mathrm{VSG}}\right.$, and $\left.Y_{\mathrm{PAH}}\right)$, relative to hydrogen, of the three dust populations. The intensity of a NIR continuum, with a temperature of $1000 \mathrm{~K}$, is also a free parameter of the model. Indeed, the inclusion of this component is required, as discussed in several studies (e.g., Lu et al. 2003), and allows us to reproduce observational data at 3.6 and $4.5 \mu \mathrm{m}$. Two different radiation fields (RFs) input models are used in this work. The strength of the field $\left(X_{\mathrm{RF}}\right)$ is a free parameter in both cases. However, for all three cases (diffuse medium, typical, and very bright $\mathrm{H}$ II regions), we impose, for dust emission associated with the atomic and molecular phases, 

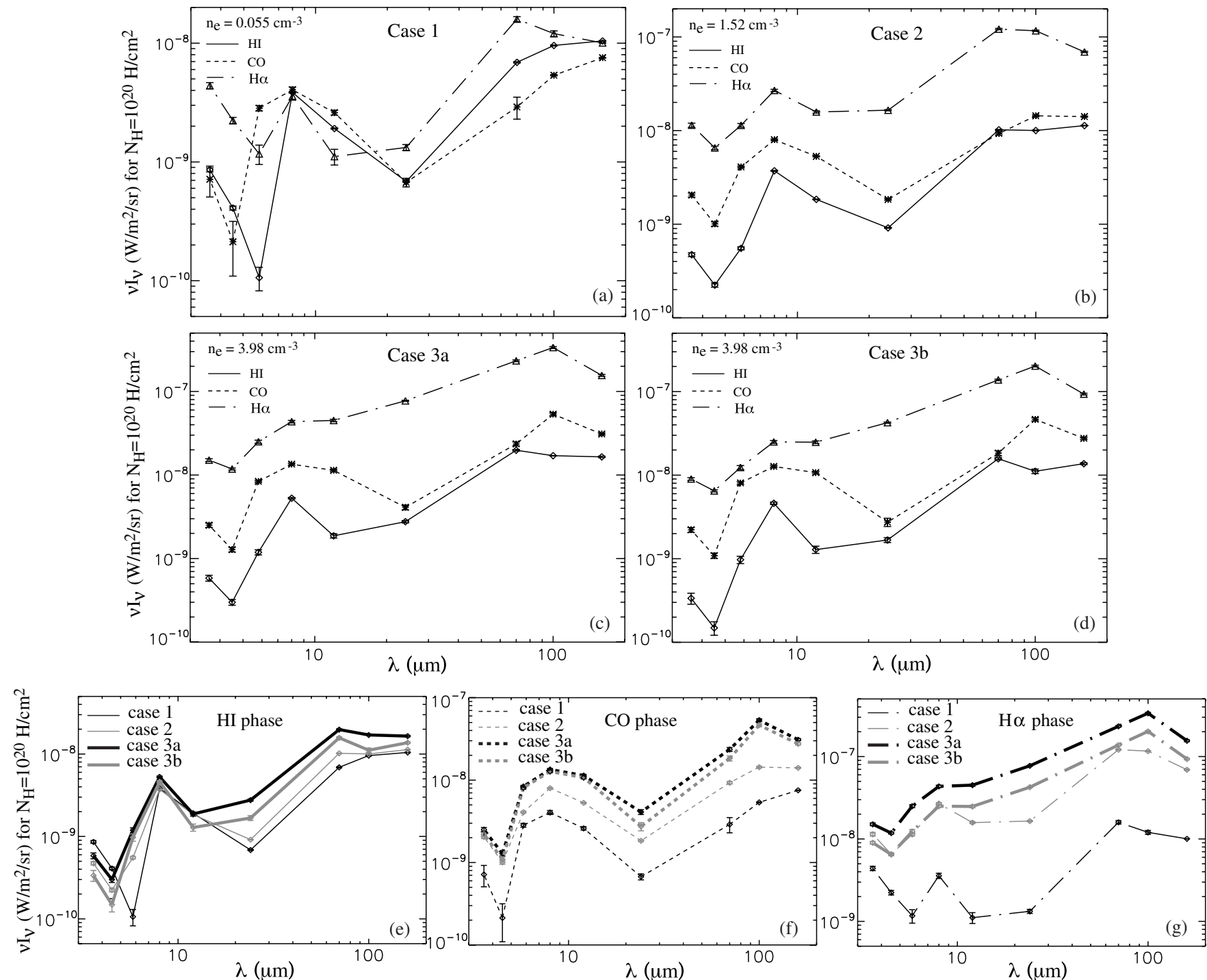

Figure 3. Spectral energy distribution associated with the atomic (solid line), molecular (dashed line), and ionized (dash-dotted line) phases of the gas, for case 1 (diffuse ionized gas, panel (a)), case 2 (typical $\mathrm{H}$ II regions, panel (b)), and case 3 (bright $\mathrm{H}$ II regions). For case 3 , we show the results of the analysis obtained by using both $\mathrm{H} \alpha$ (case 3a, panel (c)) and $4.75 \mathrm{GHz}$ data (case 3b, panel (d)). Panels (e), (f), and (g): spectral energy distributions of all cases (case 1 in thin black line, case 2 in thin gray line, case $3 \mathrm{a}$ in thick black line, and case $3 \mathrm{~b}$ in thick gray line), plotted once for each gas component (same notation as in panels (a), (b), (c), and (d)).

as well as for dust emission associated with the diffuse ionized gas in case 1, the same interstellar RF hardness as for the solar neighborhood (Mathis et al. 1983). In the following we will refer to this RF as Mathis RF. Note that, for dust in the atomic and molecular gas phases, we assume that the dominant component of the RF is always the one related to the interstellar solar neighborhood. However, for cases 2 and 3, dust in the ionized phase is associated with typical and bright $\mathrm{H}$ II regions, and therefore it is essentially heated by local hot stars. In these regions, the solar neighborhood interstellar RF is not a good approximation. Following Kawamura et al. (2009), the youngest stellar objects are less than 10 Myr. Using the GALEV code, ${ }^{6}$ which is an implementation of evolutionary synthesis models, we have generated an UV/visible spectrum of young stellar clusters of $4 \mathrm{Myr}$. In the following, we will refer to this RF as the 4 Myr cluster RF. The summary of the RFs used for each case and phase is described in Table 2, and the RF spectral shapes are shown in Figure 4.

\footnotetext{
6 Available at http://www.galev.org.
}

\subsubsection{Composite Radiation Fields Along the LOS}

Assuming a single RF component along the LOS is a simplistic hypothesis. Therefore, we tested our results using instead a combination of several RF intensities and hardnesses along the LOS. For case 1, we apply the "local-SED combination" model proposed by Dale et al. (2001) for normal star-forming galaxies. This model assumes a power-law distribution of dust mass subject to a given heating intensity $d M_{d}\left(X_{\mathrm{RF}}\right)$ :

$$
d M_{d}\left(X_{\mathrm{RF}}\right) \propto X_{\mathrm{RF}}^{-\alpha} d X_{\mathrm{RF}},
$$

where $\alpha$ controls the relative contribution of the various strengths of the field to the SEDs. Dale et al. (2001) considered RF strengths and $\alpha$ values in the range $0.3<X_{\mathrm{RF}}<10^{5}$ and $1<\alpha<2.5$, respectively. A value of $\alpha$ close to 1 indicates an active star-forming region, whereas $\alpha$ around 2.5 corresponds to the diffuse medium. This parameter is linked to the $I_{60} / I_{100}$ ratio, and therefore gives an indirect measure of dust temperature.

Here is how we proceed in our analysis. We first compute emission spectra using DustEM, for different $X_{\mathrm{RF}}$ values, and 
Results of the IR Emission Decomposition with respect to Different Gas Phases, in Units of MJy sr ${ }^{-1}$ for $10^{20} \mathrm{H} \mathrm{cm}^{-2}$, and Their $1 \sigma$ Uncertainties

\begin{tabular}{|c|c|c|c|c|c|c|c|c|c|}
\hline Cases & $\bar{I} I_{160}$ & $\overline{I 100}$ & $I_{70}$ & 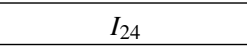 & $\overline{I I 2}$ & $I_{8}$ & $\begin{array}{l}I_{5.8} \\
\end{array}$ & $I_{4.5}$ & $I_{3.6}$ \\
\hline $1-\mathrm{HI}_{\mathrm{I}}$ & $(5.57 \pm 0.03) \times 10^{-1}$ & $(3.19 \pm 0.03) \times 10^{-1}$ & $(1.61 \pm 0.02) \times 10^{-1}$ & $(5.48 \pm 0.07) \times 10^{-3}$ & $(7.65 \pm 0.08) \times 10^{-3}$ & $(10.27 \pm 0.09) \times 10^{-3}$ & $(2.05 \pm 0.46) \times 10^{-4}$ & $(6.14 \pm 0.24) \times 10^{-4}$ & $(1.03 \pm 0.04) \times 10^{-3}$ \\
\hline $1-\mathrm{CO}$ & $(4.02 \pm 0.02) \times 10^{-1}$ & $(1.79 \pm 0.02) \times 10^{-1}$ & $(6.77 \pm 1.42) \times 10^{-2}$ & $(5.38 \pm 0.45) \times 10^{-3}$ & $(1.04 \pm 0.05) \times 10^{-2}$ & $(1.08 \pm 0.06) \times 10^{-2}$ & $(5.48 \pm 0.29) \times 10^{-3}$ & $(3.19 \pm 1.55) \times 10^{-4}$ & $(8.58 \pm 2.49) \times 10^{-4}$ \\
\hline $1-\mathrm{H} \alpha$ & $(5.36 \pm 0.02) \times 10^{-1}$ & $(4.01 \pm 0.22) \times 10^{-1}$ & $(3.72 \pm 0.18) \times 10^{-1}$ & $(1.06 \pm 0.06) \times 10^{-2}$ & $(4.44 \pm 0.67) \times 10^{-3}$ & $(9.54 \pm 0.69) \times 10^{-3}$ & $(2.26 \pm 0.42) \times 10^{-3}$ & $(3.35 \pm 0.21) \times 10^{-3}$ & $(5.27 \pm 0.32) \times 10^{-3}$ \\
\hline $2-\mathrm{HI}_{\mathrm{I}}$ & $(6.03 \pm 0.03) \times 10^{-1}$ & $(3.34 \pm 0.03) \times 10^{-1}$ & $(2.38 \pm 0.02) \times 10^{-1}$ & $(7.31 \pm 0.07) \times 10^{-3}$ & $(7.37 \pm 0.07) \times 10^{-3}$ & $(9.92 \pm 0.08) \times 10^{-3}$ & $(1.07 \pm 0.04) \times 10^{-3}$ & $(3.36 \pm 0.17) \times 10^{-4}$ & $(5.67 \pm 0.27) \times 10^{-4}$ \\
\hline $2-\mathrm{CO}$ & $(7.52 \pm 0.11) \times 10^{-1}$ & $(4.79 \pm 0.11) \times 10^{-1}$ & $(2.17 \pm 0.08) \times 10^{-1}$ & $(1.47 \pm 0.03) \times 10^{-2}$ & $(2.12 \pm 0.03) \times 10^{-2}$ & $(2.13 \pm 0.04) \times 10^{-2}$ & $(7.89 \pm 0.18) \times 10^{-3}$ & $(1.51 \pm 0.08) \times 10^{-3}$ & $(2.46 \pm 0.12) \times 10^{-3}$ \\
\hline $2-\mathrm{H} \alpha$ & $3.69 \pm 0.06$ & $3.88 \pm 0.06$ & $2.83 \pm 0.05$ & $(1.32 \pm 0.02) \times 10^{-1}$ & $(6.32 \pm 0.18) \times 10^{-2}$ & $(7.16 \pm 0.20) \times 10^{-2}$ & $(2.20 \pm 0.10) \times 10^{-2}$ & $(9.81 \pm 0.42) \times 10^{-3}$ & $(1.37 \pm 0.07) \times 10^{-2}$ \\
\hline$\overline{3 a-H I}$ & $(8.80 \pm 0.17) \times 10^{-1}$ & $(5.68 \pm 0.02) \times 10^{-1}$ & $(4.62 \pm 0.10) \times 10^{-1}$ & $(2.21 \pm 0.07) \times 10^{-2}$ & $(7.49 \pm 0.44) \times 10^{-3}$ & $(1.41 \pm 0.03) \times 10^{-2}$ & $(2.30 \pm 0.16) \times 10^{-3}$ & $(4.50 \pm 0.36$ & $(7.00 \pm 0.57) \times 10^{-4}$ \\
\hline $3 \mathrm{a}-\mathrm{CO}$ & $1.65 \pm 0.05$ & $1.78 \pm$ & $(5.50 \pm 0.30) \times 10^{-1}$ & $(3.28 \pm 0.22)$ & $(4.55 \pm 0.15$ & $(3.59 \pm 0.1$ & $(1.62 \pm 0.05$ & $(1.92 \pm 0.1$ & $(3.01 \pm 0.18) \times 10^{-3}$ \\
\hline $3 \mathrm{a}-\mathrm{H} \alpha$ & $8.30 \pm 0.22$ & $11.19 \pm 0.29$ & $5.43 \pm 0.13$ & $(6.18 \pm 0.09) \times 10^{-1}$ & $(1.80 \pm 0.06) \times 10^{-1}$ & $(1.16 \pm 0.04) \times 10^{-1}$ & $(4.84 \pm 0.21) \times 10^{-2}$ & $(1.77 \pm 0.05) \times 10^{-2}$ & $(1.81 \pm 0.07) \times 10^{-2}$ \\
\hline$\overline{3 b-H ~ I ~}$ & $(7.32 \pm 0.18) \times 10^{-1}$ & $(3.71 \pm 0.23) \times 10^{-1}$ & $(3.67 \pm 0.11) \times 10^{-1}$ & $(1.34 \pm 0.09) \times 10^{-2}$ & $(5.14 \pm 0.51) \times 10^{-3}$ & $(1.23 \pm 0.04) \times 10^{-2}$ & $(1.88 \pm 0.19) \times 10^{-3}$ & $(2.23 \pm 0.41) \times 10^{-4}$ & $(4.03 \pm 0.61) \times 10^{-4}$ \\
\hline $3 b-\mathrm{CO}$ & $1.47 \pm 0.05$ & $1.55 \pm 0.07$ & $(4.28 \pm 0.30) \times 10^{-1}$ & $(2.19 \pm 0.25) \times 10^{-2}$ & $(4.29 \pm 0.15) \times 10^{-2}$ & $(3.39 \pm 0.11) \times 10^{-2}$ & $(1.56 \pm 0.06) \times 10^{-2}$ & $(1.63 \pm 0.12) \times 10^{-3}$ & $(2.66 \pm 0.18) \times 10^{-3}$ \\
\hline 3b-radio & $4.98 \pm 0.12$ & $6.74 \pm 0.16$ & $3.22 \pm 0.07$ & $(3.40 \pm 0.06) \times 10^{-1}$ & $(9.92 \pm 0.36) \times 10^{-2}$ & $(6.67 \pm 0.26) \times 10^{-2}$ & $(2.39 \pm 0.13) \times 10^{-2}$ & $(9.73 \pm 0.28) \times 10^{-3}$ & $(1.08 \pm 0.04) \times 10^{-2}$ \\
\hline
\end{tabular}

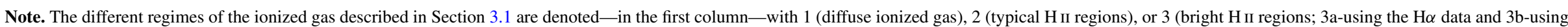
the radio data at $4.75 \mathrm{GHz}$ ). 
Table 2

Results of the Modeling Obtained by Assuming a Single Radiation Field Along the LOS

\begin{tabular}{|c|c|c|c|c|c|c|}
\hline $\mathrm{RF}$ & Cases & $\begin{array}{c}Y_{\text {tot }}(\mathrm{D} / \mathrm{G}) \\
10^{-3}\end{array}$ & $\begin{array}{c}\text { NIR cont } \\
10^{-4}\end{array}$ & $X_{\mathrm{RF}}$ & $\begin{array}{c}Y_{\mathrm{PAH}} / Y_{\mathrm{BG}} \\
10^{-2}\end{array}$ & $\begin{array}{c}Y_{\mathrm{VSG}} / Y_{\mathrm{BG}} \\
10^{-2}\end{array}$ \\
\hline Mathis & $1-\mathrm{H} \mathrm{I}_{\mathrm{I}}$ & $1.82 \pm 0.04$ & $1.98 \pm 0.22$ & $1.51 \pm 0.03$ & $4.29 \pm 0.20$ & $5.37 \pm 0.28$ \\
\hline Mathis & $1-\mathrm{CO}$ & $3.19 \pm 0.87$ & $4.90 \pm 1.36$ & $0.61 \pm 0.16$ & $9.87 \pm 5.19$ & $6.65 \pm 3.78$ \\
\hline Mathis & $1-\mathrm{H} \alpha$ & $0.50 \pm 0.07$ & $36.71 \pm 1.83$ & $6.46 \pm 0.90$ & $1.50 \pm 0.54$ & $9.47 \pm 2.80$ \\
\hline Mathis & 2-H I & $1.62 \pm 0.03$ & $1.02 \pm 0.15$ & $1.86 \pm 0.03$ & $4.00 \pm 0.14$ & $7.08 \pm 0.26$ \\
\hline Mathis & $2-\mathrm{CO}$ & $2.77 \pm 0.17$ & $14.44 \pm 0.69$ & $1.42 \pm 0.08$ & $8.94 \pm 1.07$ & $10.55 \pm 1.35$ \\
\hline 4 Myr cluster & $2-\mathrm{H} \alpha$ & $3.16 \pm 0.15$ & $91.69 \pm 3.83$ & $0.39 \pm 0.02$ & $0.88 \pm 0.09$ & $6.88 \pm 0.66$ \\
\hline Mathis & 3a-H I & $1.29 \pm 0.08$ & $3.06 \pm 0.33$ & $3.93 \pm 0.24$ & $3.27 \pm 0.41$ & $13.81 \pm 1.90$ \\
\hline Mathis & $3 \mathrm{a}-\mathrm{CO}$ & $3.61 \pm 0.35$ & $16.47 \pm 1.07$ & $2.79 \pm 0.26$ & $6.38 \pm 1.20$ & $9.13 \pm 2.10$ \\
\hline 4 Myr cluster & $3 \mathrm{a}-\mathrm{H} \alpha$ & $12.67 \pm 0.94$ & $157.06 \pm 4.41$ & $0.21 \pm 0.02$ & $0.77 \pm 0.12$ & $17.35 \pm 2.61$ \\
\hline Mathis & 3b-H I & $1.10 \pm 0.10$ & $0.78 \pm 0.37$ & $3.66 \pm 0.30$ & $3.54 \pm 0.59$ & $10.28 \pm 2.08$ \\
\hline Mathis & $3 b-C O$ & $3.34 \pm 0.36$ & $13.77 \pm 1.12$ & $2.65 \pm 0.28$ & $6.83 \pm 1.45$ & $6.26 \pm 1.95$ \\
\hline 4 Myr cluster & 3b-Radio (5 GHz) & $7.33 \pm 0.51$ & $87.89 \pm 2.65$ & $0.22 \pm 0.01$ & $0.68 \pm 0.10$ & $15.48 \pm 2.20$ \\
\hline
\end{tabular}

Notes. Three different regimes of the ionized gas are considered: diffuse (1), typical H II regions (2) and bright H II regions (3a corresponds to $\mathrm{H} \alpha$ data and $3 \mathrm{~b}$ to radio data at $4.75 \mathrm{GHz}$ ). The first column denotes the adopted radiation field spectrum: the Mathis RF or the $4 \mathrm{Myr}$ cluster Galev RF (see Section 3.3.1).

the Mathis $\mathrm{RF}$ (noted $I_{v}^{\bmod }\left(X_{\mathrm{RF}}, \mathrm{RF}_{\odot}\right)$ ). Then we sum up these emission spectra over the same $X_{\mathrm{RF}}$ range as proposed by Dale et al. (2001) according to

$$
I_{v}^{\text {tot }}=\frac{\sum_{i} \sum_{j} I_{v}^{\bmod }\left(X_{\mathrm{RF}, i}, \mathrm{RF}_{\odot}\right) \times X_{\mathrm{RF}, i}^{-\alpha_{j}}}{\sum_{i} \sum_{j} X_{\mathrm{RF}, i}^{-\alpha_{j}}} .
$$

For the atomic and molecular phases in cases 2 and 3, we check the impact of adopting a combination of the Mathis RF and the simulated 4 Myr cluster Galev RF. Equation (9) becomes

$$
\begin{aligned}
I_{v}^{\mathrm{tot}}= & \frac{\frac{1}{2} \sum_{i} \sum_{j} I_{v}^{\bmod }\left(X_{\mathrm{RF}, i}, \mathrm{RF}_{\odot}\right) \times X_{\mathrm{RF}, i}^{-\alpha_{j}}}{\sum_{i} \sum_{j} X_{\mathrm{RF}, i}^{-\alpha_{j}}} \\
& +\frac{\frac{1}{2} f \sum_{i} \sum_{j} I_{v}^{\bmod }\left(X_{\mathrm{RF}, i}, \mathrm{RF}_{\mathrm{Galev}}\right) \times X_{\mathrm{RF}, i}^{-\alpha_{j}}}{\sum_{i} \sum_{j} X_{\mathrm{RF}, i}^{-\alpha_{j}}} .
\end{aligned}
$$

The coefficient $f$ allows us to normalize the emission spectra computed with each RF hardness. Indeed, the same $X_{\mathrm{RF}}$ will not give the same absolute emission level depending on the RF used. To determine $f$, we adjust the emission spectra using the two RFs (Mathis RF and 4 Myr cluster RF) and look for the coefficient allowing the same brightness at the maximum emission. This coefficient $f$ is re-derived for each $X_{\mathrm{RF}}$. For the ionized phase in cases 2 and 3, we apply Equation (9), but replacing the Mathis RF by the 4 Myr cluster RF.

The DustEM code does not allow us to perform a model fit using a combination of RFs. Therefore, we have to use a different method to analyze the effect of a composite RF. Following Paradis et al. (2009b), in order to derive mass abundances for each dust grain species, we pre-compute the IR brightnesses associated with each gas phase, using the Galactic abundances obtained by Désert et al. (1990), for different $\alpha$ values. We then compare the observed brightnesses between 8 and $160 \mu \mathrm{m}$ with the predicted values, and infer the dust mass abundances minimizing the $\chi^{2}$ for each value of $\alpha$. We select the solution with the lowest $\chi^{2}$. The additional NIR continuum is not taken into account to compute the IR brightnesses, since we do not know the origin of this component, nor how to treat it in the case of a combination of RFs. For this reason, we do not consider IR data below $8 \mu \mathrm{m}$. The summary of the RF combinations used for each case and phase is given in Table 3 .

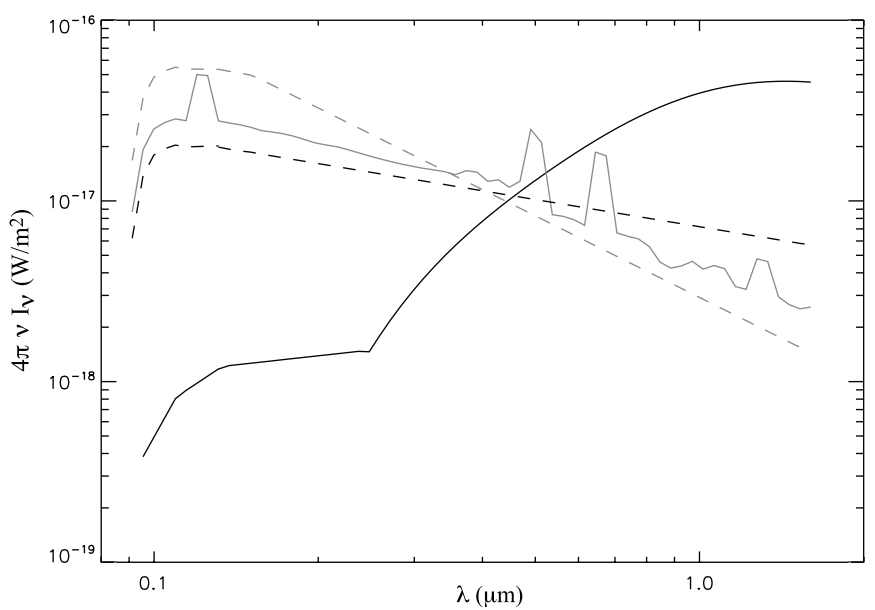

Figure 4. Radiation field templates used in this work: Mathis (solid black line), 4 Myr cluster Galev (solid gray line), and 4 Myr cluster Galev (gas lines removed) with a hardness proportional to $\lambda^{-0.5}$ and $\lambda^{-1.5}$ (dashed black and dashed gray lines).

\section{RESULTS AND DISCUSSION}

The dust emission spectra are shown in Figure 3. Figure 5 illustrates the SED modeling obtained using DustEM, assuming a single RF component. In the following we analyze the results for the diffuse ionized gas, and typical and bright $\mathrm{H}$ II regions. We also discuss their consistency using the hypothesis of an RF combination. The best-fit parameters for each phase of the gas (molecular, atomic, and ionized) and each regime of the ionized gas, as well as for different RFs assumptions, are provided in Tables 2 and 3.

\subsection{Single Radiation Field Component \\ 4.1.1. Case 1: Diffuse Ionized Gas}

Figures 3 (panel (a)) and 5 indicate that, when compared to the atomic and molecular phases, dust emission associated with the ionized gas phase has a relevant contribution to the total emission. However, we emphasize that the absolute amplitude of the spectrum for the ionized phase is proportional to $n_{\mathrm{e}}$. As a consequence, if $n_{\mathrm{e}}$ has been underestimated/ overestimated, the absolute level of the spectrum has also been 
Table 3

Results of the Modeling Obtained by Assuming a Composite Radiation Field Along the LOS

\begin{tabular}{lcrccc}
\hline \hline \multicolumn{1}{c}{ RF } & Cases & $\begin{array}{c}Y_{\text {tot }}(\mathrm{D} / \mathrm{G}) \\
10^{-3}\end{array}$ & $\alpha$ & $\begin{array}{c}Y_{\mathrm{PAH}} / Y_{\mathrm{BG}} \\
10^{-2}\end{array}$ & $\begin{array}{c}Y_{\mathrm{VSG}} / Y_{\mathrm{BG}} \\
10^{-2}\end{array}$ \\
\hline$\sum_{\mathrm{i}} \sum_{\mathrm{j}}$ Mathis & $1-\mathrm{H} \mathrm{I}$ & $3.39 \pm 0.02$ & 2.47 & $4.57 \pm 0.06$ & $2.11 \pm 0.08$ \\
$\sum_{\mathrm{i}} \sum_{\mathrm{j}}$ Mathis & $1-\mathrm{CO}$ & $2.35 \pm 0.13$ & 2.50 & $8.68 \pm 0.78$ & $4.17 \pm 0.91$ \\
$\sum_{\mathrm{i}} \sum_{\mathrm{j}}$ Mathis & $1-\mathrm{H} \alpha$ & $3.06 \pm 0.12$ & 2.29 & $2.74 \pm 0.28$ & $0.43 \pm 0.50$ \\
\hline$\sum_{\mathrm{i}} \sum_{\mathrm{j}}$ Mathis + 4 Myr cluster & $2-\mathrm{H} \mathrm{I}$ & $6.23 \pm 0.03$ & 2.50 & $2.42 \pm 0.03$ & $1.56 \pm 0.05$ \\
$\sum_{\mathrm{i}} \sum_{\mathrm{j}}$ Mathis + 4 Myr cluster & $2-\mathrm{CO}$ & $7.83 \pm 0.11$ & 2.50 & $4.91 \pm 0.12$ & $3.93 \pm 0.22$ \\
$\sum_{\mathrm{i}} \sum_{\mathrm{j}}$ 4 Myr cluster & $2-\mathrm{H} \alpha$ & $1.81 \pm 0.02$ & 2.50 & $0.88 \pm 0.03$ & $1.02 \pm 0.09$ \\
$\sum_{\mathrm{i}} \sum_{\mathrm{j}}$ Mathis + 4 Myr cluster & $3 \mathrm{a}-\mathrm{H} \mathrm{I}$ & $8.38 \pm 0.16$ & 2.32 & $1.66 \pm 0.07$ & $0.27 \pm 0.26$ \\
$\sum_{\mathrm{i}} \sum_{\mathrm{j}}$ Mathis + 4 Myr cluster & $3 \mathrm{a}-\mathrm{CO}$ & $19.38 \pm 0.58$ & 2.50 & $3.38 \pm 0.17$ & $3.81 \pm 0.55$ \\
$\sum_{\mathrm{i}} \sum_{\mathrm{j}}$ 4 Myr cluster & $3 \mathrm{a}-\mathrm{H} \alpha$ & $3.53 \pm 0.08$ & 2.50 & $0.76 \pm 0.04$ & $8.39 \pm 0.39$ \\
$\sum_{\mathrm{i}} \sum_{\mathrm{j}}$ Mathis + 4 Myr cluster & $3 \mathrm{~b}-\mathrm{H} \mathrm{I}$ & $7.17 \pm 0.19$ & 2.38 & $1.89 \pm 0.10$ & $0.72 \pm 0.41$ \\
$\sum_{\mathrm{i}} \sum_{\mathrm{j}}$ Mathis + 4 Myr cluster & $3 \mathrm{~b}-\mathrm{CO}$ & $17.01 \pm 0.59$ & 2.50 & $3.61 \pm 0.20$ & $2.08 \pm 0.64$ \\
$\sum_{\mathrm{i}} \sum_{\mathrm{j}}$ 4 Myr cluster & 3b-Radio $(5 \mathrm{GHz})$ & $2.16 \pm 0.04$ & 2.50 & $0.71 \pm 0.04$ & $7.00 \pm 0.36$ \\
\hline
\end{tabular}

Notes. Three different regimes of the ionized gas are considered: diffuse (1), typical H II regions (2), and bright $\mathrm{H}$ II regions ( $3 \mathrm{a}$ corresponds to $\mathrm{H} \alpha$ data and $3 \mathrm{~b}$ to radio data at $4.75 \mathrm{GHz}$ ). The first column denotes the adopted composite radiation field spectrum: the Mathis RF or the 4 Myr cluster Galev RF (see Section 3.3.2).

underestimated/overestimated, although the spectral shape will remain unchanged. Here, as discussed in Section 3.1, we take $n_{\mathrm{e}}$ equal to $0.055 \mathrm{~cm}^{-3}$, which is the average observed value in our Galaxy, as reported by Haffner et al. (2009).

In this case, the adopted RF hardness is the same for all gas phases, as discussed in Section 3.3.1. Therefore, it is possible to perform a direct comparison among the temperature estimates resulting from the best-fit RF intensities. We remind the reader that if we assume a graybody dust emission model with spectral index $\beta$, then the RF intensity and the equilibrium temperature of the BGs are related through

$$
\frac{X_{\mathrm{RF}}^{\mathrm{LMC}}}{X_{\mathrm{RF}}^{\odot}}=\left(\frac{T_{d}^{\mathrm{LMC}}}{T_{d}^{\odot}}\right)^{4+\beta}
$$

where $X_{\mathrm{RF}}^{\odot}$ is the RF scaling factor for the Mathis $\mathrm{RF}\left(X_{\mathrm{RF}}^{\odot} \simeq 1\right)$, and the dust temperature in the solar neighborhood $T_{d}^{\odot}$ is taken equal to $17.5 \mathrm{~K}$ (Boulanger et al. 1996), with $\beta=2$. We find dust temperatures equal to $18.7 \mathrm{~K}, 16.1 \mathrm{~K}$, and $23.9 \mathrm{~K}$ for the atomic, molecular, and ionized phases, respectively. These results confirm that dust associated with the ionized phase is warmer than dust in the atomic and molecular gas phases, although our derived temperature for the diffuse $\mathrm{H}^{+}$is slightly lower than the value obtained by Paladini et al. (2007), of $26.6 \pm$ $0.1 \mathrm{~K}$. As for dust emissivity for the ionized phase, we obtain an estimate of $2.3 \times 10^{-26} \mathrm{~cm}^{2} \mathrm{H}^{-1}$ at $160 \mu \mathrm{m}$. For comparison, Lagache et al. (2000) find, at high latitude, an emissivity of $1.1 \times 10^{-25} \mathrm{~cm}^{2} \mathrm{H}^{-1}$ at $250 \mu \mathrm{m}$, which corresponds to an emissivity of $2.7 \times 10^{-25} \mathrm{~cm}^{2} \mathrm{H}^{-1}$ at $160 \mu \mathrm{m}$ assuming a spectral index equal to 2, while Paladini et al. (2007), for the Galactic plane, report a value of $35.1 \mathrm{MJy} \mathrm{sr}^{-1}$ for $10^{20} \mathrm{H} \mathrm{cm}^{-2}$ at $140 \mu \mathrm{m}$, corresponding to $8.7 \times 10^{-25} \mathrm{~cm}^{2} \mathrm{H}^{-1}$ at $160 \mu \mathrm{m}$. Therefore, even if in terms of temperature, dust in the ionized phase of the LMC is roughly comparable to its Galactic plane counterpart, it appears to be 40 times less emissive in the LMC with respect to the Galactic plane, and 10 times less emissive with respect to the high Galactic latitudes. The low value in the LMC cannot be only due to the low metallicity of the LMC ( $Z \simeq 0.3-0.5 Z_{\odot}$; Westerlund 1997) with respect to our Galaxy, as the discrepancy in emissivity is significantly larger than the difference in metallicity. For the Galactic plane, at least part of the difference could be induced by confusion along the LOS.
However, we think that different dust properties in the ionized gas phase of the LMC compared to our Galaxy (high latitudes and Galactic plane) are likely at the origin of the observed discrepancy.

From Table 2, the total amount of dust in the ionized phase, similar to the dust-over-gas ratio (D/G), appears to be 3.6 and 6.4 times lower than in the atomic and molecular phases. In particular, the PAH relative abundance with respect to BGs reveals a decrement by a factor of 2.9 and 6.6 when compared to the atomic and molecular phases, respectively. The high value of PAH relative abundance in the molecular phase is connected to the low $X_{\mathrm{RF}}$ which, in turn, is a consequence of the shielding effect from the RF induced by the higher density characterizing this phase of the gas. For the VSG relative abundance in the ionized phase, we do not find the same depletion behavior as for PAHs. The VSG relative abundance is in fact roughly the same in the three phases. We interpret this effect as VSGs being resilient to the RF.

Remarkably, the spectrum of dust associated with the ionized phase highlights more emission in the NIR domain, by a factor of 7.5 and 18.5, compared to the molecular and atomic phases. The origin of this effect is not clear. Lu et al. (2003) argue that the NIR continuum intensity is quite linear with the strength of the aromatic features in emission. They suggest that both this continuum and the aromatic features originate from similar carriers. Flagey et al. (2006), using Spitzer data for the Galactic diffuse interstellar medium (ISM), require the presence of a continuum to explain the observations, but they conclude that the continuum carriers might not be the PAHs. What we observe in the ionized phase of the LMC is a decrease of the PAH relative abundance and an increase of the NIR continuum, whereas the low value of the NIR continuum intensity in the molecular phase is associated with a high value of PAH relative abundance. Therefore our results favor Flagey et al.'s (2006) interpretation.

\subsubsection{Case 2: Typical H II Regions}

In this case, the modeling seems to reveal (Figure 3, panel (b)) that emission associated with the ionized gas phase is not only a major contributor to the total emission, but is even dominant with respect to the other components, i.e., atomic and molecular. Nevertheless, as in the previous section, we need to remind the 

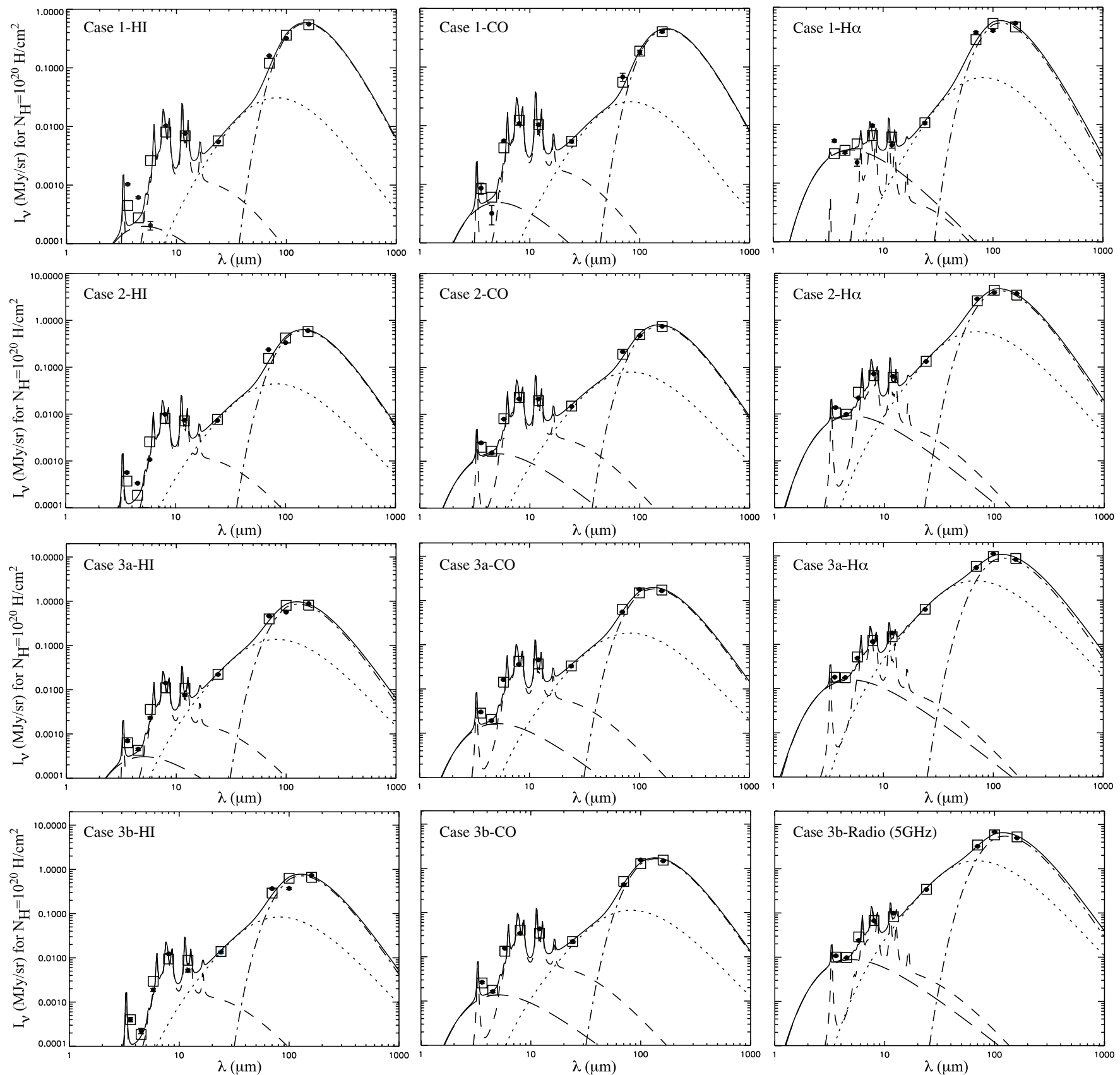

Figure 5. Results of the fits obtained with the dust emission model (DustEM) assuming a single RF along the LOS, for each gas phase and for each regime of the ionized gas: diffuse (case 1), typical $\mathrm{H}_{\mathrm{II}}$ regions (case 2), and bright $\mathrm{H}$ II regions (case 3). In particular: case 3 a corresponds to $\mathrm{H} \alpha$ data, case $3 \mathrm{~b}$ to radio data. The squares show the fits of the model after applying the color correction in each Spitzer and IRIS bands. Different curves denote the contributions from various grain populations: total (solid), BG (dot-dash), VSG (dot), PAH (dash), and the NIR continuum (long dash).

reader that this result quite strongly depends on the electron density. At the same time, we do not think that the adopted value can be off by a large amount, given that it has been derived from the Hil regions in the HK86 catalog. In fact, we should be wrong by a factor 5-6 in order for the ionized gas spectrum to match the atomic and molecular spectra at $160 \mu \mathrm{m}$.

For the ionized phase associated with typical H II regions, we adopt-as discussed in Section 3.3.1-a bluer RF input model. Indeed, the Mathis RF is not representative in this case, as the ionized gas is mostly heated by radiation from nearby stars. Since we assume different RFs for different gas phases, we cannot directly compare the temperature inferred from the model. However, Figure 5 shows evidence of a shift of the BG emission peak toward shorter wavelengths in the ionized gas phase compared to the other phases. This result would suggest an increase of dust temperature when going from the atomic or molecular phase to the ionized one. The hardness of the RF modeled with Galev is proportional to $\lambda^{-1}$. We have checked our results against different slopes of the input RF, for example $\lambda^{-0.5}$ and $\lambda^{-1.5}$ (see Figure 4), and verified that the changes in the outputs are negligible.

As for case 1, the ionized phase appears to be characterized by less PAHs relative to BGs. In particular, the PAH relative abundance decreases by a factor $4.5-10$, compared to the relative abundance in the atomic and molecular phases. This is likely a consequence of a higher RF intensity with respect to the other phases. 
Furthermore, in a similar fashion to what we find for the diffuse ionized medium, the molecular phase presents an enhancement of the PAH relative abundance. For typical H II regions, the $\mathrm{CO}$ data trace the photodissociation region (PDR) surrounding the ionized gas, where the local high density contributes to the shielding of the PAHs from the stellar RF. Interestingly, the atomic phase also shows a decrease in the PAH relative abundance compared to the molecular phase, even if the effect is less important than for the ionized phase. The atomic phase is subject to an attenuated stellar RF, as well as to moderate radiation from the ISM. The combined effect of both RFs might cause the partial PAH destruction, or a decrease of the excitation state of the PAHs. In this latter scenario, the PAHs would be less emissive. Overall, the results obtained for typical $\mathrm{H}$ II regions confirm those derived for the diffuse $\mathrm{H}^{+}$. The VSG relative abundance is qualitatively the same in the three phases.

As for case 1, we note that the NIR continuum intensity is increased in the ionized phase with respect to the atomic phase, in this case by a factor of 90 , whereas the PAH relative abundance decreases. This result corroborates the hypothesis that the NIR continuum is not correlated with the PAH abundance.

\subsubsection{Case 3: Bright H II Regions}

In case 3, we are looking at dust emission in the vicinity of conspicuous massive star associations. As in the previous case, dust emission in the ionized phase appears to dominate the total emission (see Figure 3, panels (c) and (d)), although with the caveat discussed in Sections 4.1.1 and 4.1.2.

Results derived using $\mathrm{H} \alpha$ and radio data are in good agreement. The lower amplitude of the spectrum associated with the ionized phase when we make use of the Parkes rather than the SHASSA data in Equation (6) is likely partly a consequence of dust absorption in the SHASSA map, as already mentioned in Section 2.2.3.

From Table 2, we note that the RF intensity and the dust relative abundances are in good agreement for both the ionized gas tracers. The results show, for the ionized gas phase in particular, a low PAH relative abundance comparable to case 2 , and an increase of the VSG relative abundance by a factor of $2.2-2.5\left(Y_{\mathrm{VSG}} / Y_{\mathrm{BG}} \simeq 6.9\right.$ in case 2 and $Y_{\mathrm{VSG}} / Y_{\mathrm{BG}} \simeq 15.5-17.4$ in cases 3). Remarkably, these conclusions do not depend on the specific spectral shape of the RF modeled with Galev. The molecular phase appears again to be characterized by a high $\mathrm{PAH}$ relative abundance. We note that the PAH destruction in the ionized phase becomes more pronounced going from case 1 to case 3, probably due to the increase of the RF intensity from the diffuse medium to bright $\mathrm{H}$ in regions. This is consistent with previous findings reported in the literature. For instance, using ISOCAM data, Contursi et al. (2007) found a lack of aromatic feature carriers in the core of the LMC HII region N4, and suggested that the high and hard RF might be responsible for the PAH destruction. Likewise, Povich et al. (2007) identified $\mathrm{PAH}$ destruction at the edge of the $\mathrm{H}$ II region M17. Boulanger et al. (1988) explained the decrease of the $12 \mu \mathrm{m}$ emission in the California Nebula by grain destruction in regions with high UV radiation. They estimated that around $80 \%$ of the $12 \mu \mathrm{m}$ emitters could be destroyed whenever the energy density is larger than 50 times the energy density in the solar neighborhood. Despite this theoretical and observational evidence, we cannot state that the RF is systematically responsible for the depletion of PAHs in $\mathrm{H}$ II regions. Indeed, several other processes could be at work, like Coulomb destruction of PAH dications or dehydrogenation by direct photodissociation, as suggested by Bernard et al.
(1994) based on the analysis of the $3.3 \mu \mathrm{m}$ emission and nearby continuum in the planetary nebula BD+30 3639. Moreover, Giard et al. (1994) studied the same dust feature in the Orion bar and M17 and proposed that $\mathrm{H}^{+}$chemical sputtering could cause PAH destruction.

In terms of temperature, as in case 2 , we can deduce from Figure 5 the presence of warmer dust in the ionized gas as compared to the other phases, from the fact that the peak of emission for the BGs is located at shorter wavelengths. Finally, as for cases 1 and 2, the NIR continuum is higher in the ionized phase than in the other phases.

\subsection{Composite Radiation Fields}

In this section, we discuss the use of a combination of RFs, instead of a single RF, for fitting the SEDs corresponding to each phase of the gas (see Table 3 ).

For case 1, the values given in Table 3 confirm the decrease of the PAH relative abundance in the ionized phase, as evidenced in Section 4.1.1. On the contrary, the enhancement of the PAHs in the molecular phase might suggest that these environmental conditions are indeed particularly favorable for their survival. For the VSG relative abundance in the ionized phase, we find a significantly lower value with respect to what we obtain by adopting a single RF component (see Tables 2 and 3). However, the VSG relative abundance is highly dependent on the quality of the fit at FIR wavelengths and, in the ionized phase, the model exhibits an important lack of emission at $70 \mu \mathrm{m}$, as well as a higher $\chi^{2}$, as compared to a single RF modeling. This might indicate that the low VSG relative abundance has, for a composite RF, been underestimated.

For case 2, the combination of the Mathis with the $4 M y r$ cluster RFs, both for the atomic and molecular phases, generates a bluer RF than a single Mathis RF. Therefore, as expected, we find a decrease in the PAH and VSG relative abundances. Indeed, PAHs and VSGs absorb most of their energy at short wavelengths, while BGs absorb energy over the whole RF spectrum. As a consequence, PAHs and VSGs absorb and reemit more energy with a bluer RF, and to produce the same IR flux with a bluer RF than the Mathis RF, the model requires less PAHs and VSGs. For the ionized phase, the combination of 4 Myr cluster RFs gives the same PAH relative abundance of a single 4 Myr cluster RF, whereas a significant decrease of the VSG relative abundance is observed. However, the general trend is similar to that found for a single RF: as in case 1, the highest PAH relative abundance is in the molecular phase, which also exhibits more VSGs with respect to BGs, while the atomic phase shows intermediate values of PAH and VSG relative abundances, compared to the other gas phases.

In case 3 , as for case 2, the PAH relative abundance in the atomic and molecular phases decrease by a factor $\sim 2$ compared to a single RF. In the ionized phase, we still have less PAHs than in the atomic phase, by a factor of 2.2 and 2.7, for cases $3 \mathrm{a}$ and $3 \mathrm{~b}$, respectively, and by a factor between 4.4 and 5.1 compared to the molecular phase. Results are therefore in agreement with the modeling with a single RF.

For the VSG relative abundance, we obtain, for the atomic phase, different and contradictory results depending on the RF assumption, therefore preventing a clear interpretation of the results. In the ionized phase, the modeling confirms the enhancement of the relative VSG abundance already highlighted by a single RF.

Overall, the results obtained assuming a composite RF substantially corroborate those derived with a single RF. 


\subsection{Evolution of Dust Emission Associated with Different Gas Components}

In panels (e), (f), and (g) of Figure 3, we present the results of the analysis in a slightly different fashion, i.e., by combining in individual plots, each corresponding to a given gas phase, the SEDs obtained for all cases (1, 2, and 3). We can see that no matter which gas component we consider, the amplitude of the spectrum always increases going from the diffuse medium to bright $\mathrm{H}$ II regions. However, for the atomic phase, the difference in amplitude between the different cases is less important compared to the other gas components, and a rather remarkable agreement among cases is found at $8 \mu \mathrm{m}$. As previously noted, the absolute level of dust emission in the ionized gas is directly proportional to $n_{\mathrm{e}}$, for which the adopted value changes according to the specific environment. Therefore, the discrepancy in absolute amplitude of the spectra for that phase is not well constrained. On the contrary, for the atomic and molecular phases, we are confident that the recovered SED amplitudes are reliable, as these depend on the $\mathrm{HI}_{\mathrm{I}}$ and $\mathrm{CO}$ conversion factor (Equations (1) and (2)) which, respectively, is known to be constant for a given environment $(\mathrm{HI})$, and has been accurately determined for the majority of the clouds (CO).

We observe prominent $8 \mu \mathrm{m}$ emission in the atomic phase, probably associated with the $7.7 \mu \mathrm{m}$ PAH feature. This emission feature does not show in the ionized phase for the case of $\mathrm{H}$ II regions, likely due to $\mathrm{PAH}$ depletion. In comparison, the molecular phase is clearly characterized by strong $8 \mu \mathrm{m}$ emission, as well as by significant emission at 5.8 and $12 \mu \mathrm{m}$.

In the FIR domain, the atomic phase presents an excess of emission at $70 \mu \mathrm{m}$, as does the ionized phase in the diffuse medium. This finding is in agreement with Bernard et al. (2008).

\section{CONCLUSIONS}

Combining IR data with tracers of $\mathrm{HI}, \mathrm{H}_{2}$, and $\mathrm{H}^{+}$, we have evidence, for the first time, of dust emission associated with the ionized gas in the LMC. We have performed our analysis for different regimes of ionized gas: diffuse gas (case 1), typical H II regions (case 2), and compact/bright $\mathrm{H}$ II regions (case 3 ). We have modeled the spectra associated with the atomic, molecular, and ionized phases of the gas, using different assumptions for the RF, to test the robustness of our results. The results obtained by a combination of radiation fields along the LOS confirm the results derived by assuming a single RF.

We report a systematic warmer dust temperature in the ionized phase compared to the atomic and molecular phases. The inferred emissivity in the diffuse ionized gas is $2.3 \times$ $10^{-26} \mathrm{~cm}^{2} \mathrm{H}^{-1}$ at $160 \mu \mathrm{m}$, which is lower than the Galactic values by a factor higher than the metallicity ratio between these two galaxies. This result might suggest different properties of dust in the ionized gas of the LMC compared to our Galaxy. We also find, for all cases $(1,2,3)$, a significant decrease of the PAH relative abundance in the ionized phase compared to the other phases, with a larger difference between the phases for cases 2 and 3 . We interpret this result as due to PAH destruction, probably caused by the increased RF in the ionized phase, although the origin of this phenomenon is still under investigation.

At the same time, the molecular phase appears to favor the survival of the PAHs. In addition, when one compares bright $\mathrm{H}$ II regions with typical $\mathrm{H}_{\mathrm{II}}$ regions, the ionized phase shows an enhancement of the VSG relative abundance by more than a factor of 2 . We also find an important increase of the NIR continuum in the ionized phase with respect to the other gas phases, which does not seem to correlate directly with PAH emission. By comparing results derived using the $\mathrm{H} \alpha$ SHASSA map and the Parkes radio data, the estimated extinction in $\mathrm{H} \alpha$ for bright $\mathrm{H}$ II regions is found equal to $0.51 \mathrm{mag}$ ( or $A(V)=$ $0.63 \mathrm{mag})$.

Finally we observe, for all gas components, a systematic increase of the dust emission going from the diffuse medium to bright $\mathrm{H}$ in regions.

The origin of the destruction of the PAHs in the ionized phase (especially, in H II regions) is still not fully understood. Moreover, the depletion of this species is not a systematic effect, suggesting that changes in the $\mathrm{PAH}$ properties could also occur from one $\mathrm{H}$ II region to another. A forthcoming publication will investigate PAH depletion by means of the spectroscopic Spitzer/IRS data available in the context of the $S A G E$-Spec Legacy survey (Kemper et al. 2010). This work will allow us to study variations of the PAH emission at small angular scales within individual $\mathrm{H}$ II regions.

We thank the referee for useful comments, which helped to improve the content of the paper. We also thank M. Filipovic and A. Hughes for helpful discussions on the Parkes radio data and on the derivation of the thermal fraction. We acknowledge the use of the DustEM package. The NANTEN project is based on a mutual agreement between Nagoya University and the Carnegie Institution of Washington (CIW). This work is financially supported in part by a Grand-in-Aid for Scientific Research from JSPS (Nos. 22244014 and 30377931).

\section{REFERENCES}

Bennett, C. L., et al. 1992, ApJ, 396, L7

Bennett, C. L., et al. 2003, ApJ, 583, 1

Benoît, A., et al. 2002, Astropart. Phys., 17, 101

Bernard, J.-P., Giard, M., Normand, P., \& Tiphène, D. 1994, A\&A, 289, 524

Bernard, J.-P., et al. 2008, AJ, 136, 919

Boulanger, F., Abergel, A., \& Bernard, J.-P. 1996, A\&A, 312, 256

Boulanger, F., Beichman, C., Desert, F. X., Helou, G., Perault, M., \& Ryter, C. 1988, ApJ, 332, 328

Compiègne, M., Abergel, A., Verstraete, L., \& Habart, E. 2008, A\&A, 491, 797

Compiègne, M., et al. 2011, A\&A, 525, 103

Contursi, A., Rubio, M., Sauvage, M., Barba, D., Cesarsky, R., \& Boulanger, F. 2007, A\&A, 469, 539

Dale, D. A., Helou, G., Contursi, A., Silbermann, N. A., \& Kolhatkar, S. 2001, ApJ, 549, 215

Désert, F.-X., Boulanger, F., \& Puget, J.-L. 1990, A\&A, 237, 215

Dickinson, C., Davies, R. D., \& Davis, R. J. 2003, MNRAS, 341, 369

Dobashi, K., Bernard, J.-P., Hughes, A., Paradis, D., Reach, W. T., \& Kawamura, A. 2008, A\&A, 484, 205

Everett, J. E., \& Churchwell, E. 2010, ApJ, 713, 592

Feast, M. 1999, in IAU Symp. 190, New View of the Magellanic Clouds, ed. Y.-H. Chu et al. (San Francisco, CA: ASP), 542

Filipovic, M. D., Haynes, R. F., White, G. L., Jones, P. A., Klein, U., \& Wielebinski, R. 1995, A\&AS, 111, 311

Flagey, N., Boulanger, F., Verstraete, L., Miville-Deschênes, M. A., NoriegaCrespo, A., \& Reach, W. T. 2006, A\&AS, 453, 969

Fukui, Y., et al. 2008, ApJS, 178, 56

Gaustad, J. E., McCullough, P. R., Rosing, W., \& van Buren, D. 2001, PASP, 113,1326

Giard, M., Bernard, J. P., Lacombe, F., Normand, P., \& Rouan, D. 1994, A\&A, 291,239

Gordon, K. D., Clayton, G. C., Misselt, K. A., Landolt, A. U., \& Wolff, M. J. 2003, ApJ, 594, 279

Haffner, L. M., et al. 2009, Rev. Mod. Phys., 81, 969

Hauser, M. G. 1993, in AIP Conf. Proc. 278, Back to the Galaxy, ed. S. S. Holt \& F. Verter (Melville, NY: AIP), 201

Hughes, A., Wong, T., Ekers, R., Staveley-Smith, L., Filipovic, M., Maddison, S., Fukui, Y., \& Mizuno, N. 2006, MNRAS, 370, 363 
Hughes, A., et al. 2010, MNRAS, 406, 2065

Imara, N., \& Blitz, L. 2007, ApJ, 662, 969

Kawamura, A., et al. 2009, ApJS, 184, 1

Kemper, F., et al. 2010, PASP, 122, 683

Kennicutt, R. C., \& Hodge, P. W. 1986, ApJ, 306, 130

Kim, S., Staveley-Smith, L., Dopita, M. A., Sault, R. J., Freeman, K. C., Lee, Y., \& Chu, Y.-H. 2003, ApJS, 148, 473

Lagache, G., Abergel, A., Boulanger, F., Désert, F. X., \& Puget, J.-L. 1999, A\&A, 344, 322

Lagache, G., Haffner, L. M., Reynolds, R. J., \& Tufte, S. L. 2000, A\&A, 354, 247

Lu, N., et al. 2003, ApJ, 588, 199

Mathis, J. S., Mezger, P. G., \& Panagia, N. 1983, A\&A, 128, 212

Meinert, D., Klein, U., Xu, C., Haynes, R., \& Wielebinski, R. 1993, in Lecture Notes in Physics 416, New Aspects of Magellanic Cloud Research, ed. B. Baschek, G. Klare, \& J. Lequex (Berlin: Springer), 130

Meixner, M., et al. 2006, AJ, 132, 2268

Miville-Deschênes, M. A., \& Lagache, G. 2005, ApJS, 157, 302

Neugebauer, G., et al. 1984, ApJ, 278, L1

Niklas, S., Klein, U., \& Wielebinski, R. 1997, A\&A, 322, 19

Odegard, N., Arendt, R. G., Dwek, E., Haffner, L. M., Hauser, M. G., \& Reynolds, R. J. 2007, ApJ, 667, 11

O’Donnell, J. E. 1994, ApJ, 422, 158
Paladini, R., De Zotti, G., Davies, R., \& Giard, M. 2005, MNRAS, 360, 1545

Paladini, R., Montier, L., Giard, M., Bernard, J. P., Dame, T. M., Ito, S., \& Macias-Perez, J. F. 2007, A\&A, 465, 839

Paradis, D., Bernard, J.-Ph., \& Mény, C. 2009a, A\&A, 506, 745

Paradis, D., et al. 2009b, AJ, 138, 196

Paradis, D., et al. 2011, AJ, 141, 43

Peeters, E., Tielens, A. G. G. M., Boogert, A. C. A., Hayward, T. L., \& Allamandola, L. J. 2005, ApJ, 620, 774

Planck Collaboration 2011, A\&A, submitted (arXiv:1101.2029)

Povich, M. S., et al. 2007, ApJ, 660, 346

Reynolds, R. J., Tufte, S. L., Haffner, L. M., Jaehnig, K., \& Percival, J. W. 1998, PASA, 15,14

Spitzer, L. 1978, Physical Processes in the Interstellar Medium (New York: Wiley-Interscience)

Staveley-Smith, L., Kim, S., Calabretta, M. R., Haynes, R. F., \& Kesteven, M. J. 2003, MNRAS, 339, 87

Stepnik, B., et al. 2003, A\&A, 398, 551

van Leeuwen, F., Feast, M. W., Whitelock, P. A., \& Laney, C. D. 2007, MNRAS, 379,723

Watson, C., Corn, T., Churchwell, E. B., Babler, B. L., Povich, M. S., Meade, M. R., \& Whitney, B. A. 2009, ApJ, 694, 546

Westerlund, B. E. 1997, The Magellanic Clouds (New York: Cambridge Univ. Press) 